\title{
Automated classification of breast cancer morphology in histopathological images
}

\author{
Ville Ojansivu ${ }^{1 *}$, Nina Linder ${ }^{1}$, Esa Rahtu², Matti Pietikäinen², Mikael Lundin ${ }^{1}$ Heikki Joensuu ${ }^{3}$, Johan Lundin ${ }^{1}$ \\ From 11th European Congress on Telepathology and 5th International Congress on Virtual Microscopy \\ Venice, Italy. 6-9 June 2012
}

\section{Background}

The morphology of a breast cancer tumour, as examined through an optical microscope, is currently assessed visually by the pathologist in parallel with making the cancer diagnosis. The grade of differentiation, which describes how closely the morphology of the tumour resembles the corresponding healthy tissue of an organ, is undisputedly related to the outcome of breast cancer [1]. However, tumour grade is largely regarded as an unreliable prognostic factor due to its poor reproducibility [2]. The visually determined morphology is afflicted with a poor inter- and intra observer agreement, which prevents grade from being fully utilized as an important outcome predictor. The same pathologist may assign different grade to the same tumour when assessment is repeated, and different pathologists disagree to a substantial level when assessing the same tumour [3].

Computational diagnostic tools for estimating the morphological properties of cancer tissue would enable objective and reproducible alternative for diagnosis. This could be achieved by fully utilizing the recent advances in digital microscopy and computer vision $[4,5]$. Some attempts have already been made for automated grading of histopathological breast cancer images, but these studies have covered only limited amount of data or produce just a partial grading [6,7]. We propose a texture based algorithm for automated classification of breast cancer morphology. The method uses the recently introduced LPQ [8] as well as LBP [9] descriptors and an SVM classifier. The LPQ and LBP descriptors each form a histogram representing the statistical texture properties and have been used earlier in many texture analysis applications which include surface inspection [9], tissue analysis [5], and face recognition [8],

\footnotetext{
* Correspondence: ville.ojansivu@ee.oulu.fi

'Institute for Molecular Medicine Finland-FIMM, Helsinki, Finland
}

Full list of author information is available at the end of the article whereas SVM represents the state of the art among supervised learning based classification algorithms.

\section{Material and methods}

The image data set $(n=1092)$ was extracted from a series of digitized, whole-slide tissue microarray (TMA) samples from a nationwide cohort of breast cancer patients, FinProg [10]. A single continuous area that contains only tumor tissue was defined in each representative tissue spot in the hematoxylin-eosin (HE) stained TMA samples. The original tissue spots fit into an approximately $1600 \times 1600$ pixel image while the size of the defined square areas was varying with dimensions in the range $400-1400$ pixels. The images were scored by a human observer into three classes according to morphology: 1 (morphology resembling normal breast epithelium, extensive tubular formation, $n=182$ ), 2 (intermediate tubular formation, $\mathrm{n}=494$ ), and 3 (morphology least resembling normal breast epithelium, no tubular formation; $\mathrm{n}=416$ ). Examples of the three classes are illustrated in Figure 1.

The images were transformed to gray scale and represented by LBP [7] and LPQ [8] texture descriptors. The classification of the images into the three classes was done using three one-versus-rest SVM classifiers with a radial basis function kernel (RBF) combined with chi-square distance metric. The final class was chosen by selecting the largest of the scores produced by the individual SVM classifiers. Given the training samples and their classes, an SVM classifier learns a model for the data which aims to separate the classes in space with a margin. In testing phase, the SVM classifier assigns new data samples into the classes based on the learned model. In our experiments, the data was split into two halves for training and testing of the SVM classifiers. We did additional experiments with only the extreme class 1 and 3 samples. In this 

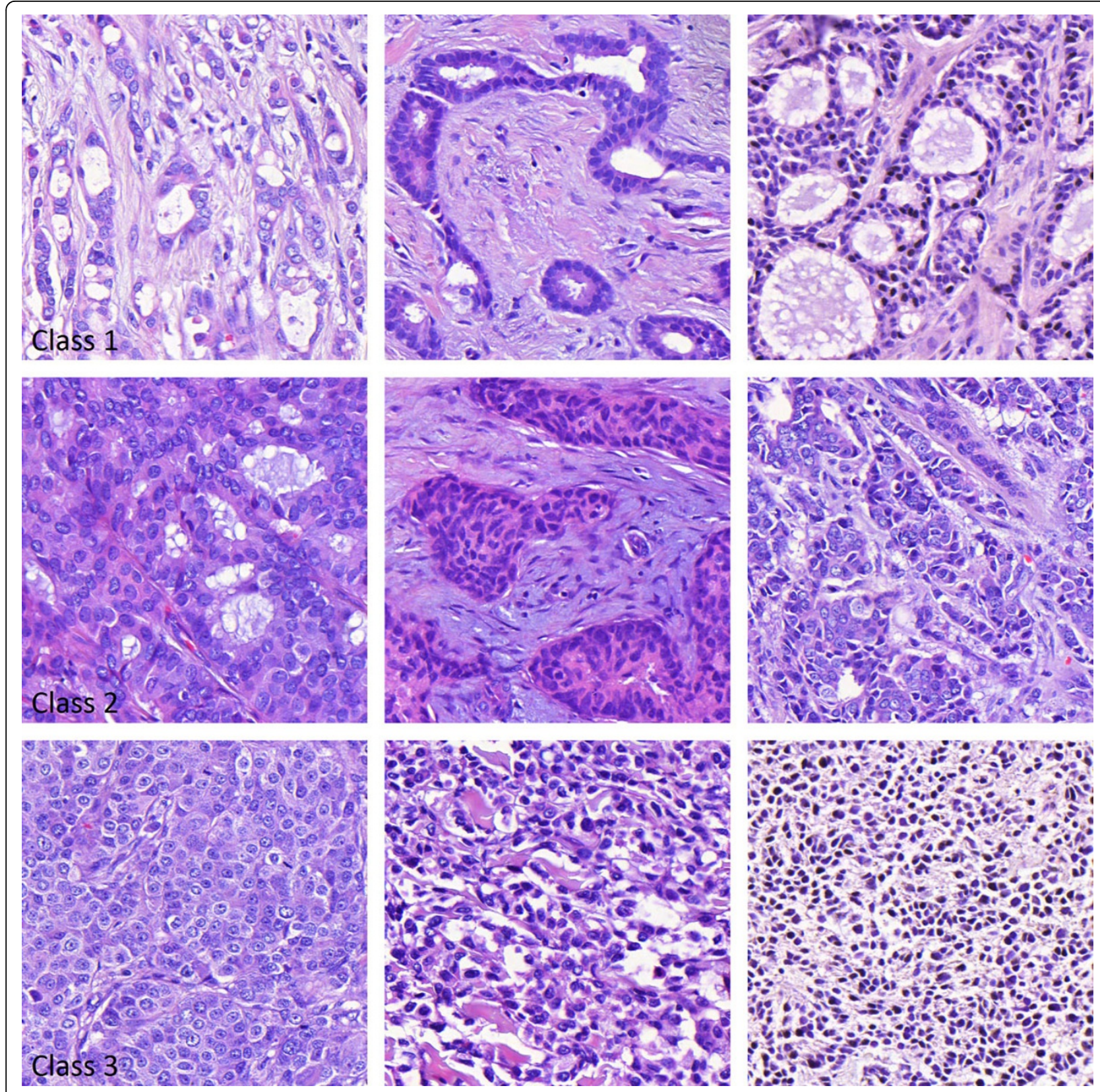

Figure 1 Examples of tissue images from three morphological classes Class 1 (top row): morphology resembling normal breast epithelium, extensive tubular formation, $n=182$; Class 2 (center row): intermediate tubular formation, $n=494$; and Class 3 (bottom row): morphology least resembling normal breast epithelium, no tubular formation, $n=416$. Images are classified into the three classes by a human observer.

case, we used the same descriptors and a binary SVM classifier with an RBF kernel.

\section{Results and discussion}

The experiments were performed using different combinations of LBP and LPQ descriptor variants as well as by various scales of the images. The best classification results were achieved by combining the basic versions of LPQ and LBP descriptors with radius $r=1$ and number of samples $\mathrm{p}=8$ into a 512-dimensional feature vector and using the original image scale 1:1. The receiver operating characteristic (ROC) curves illustrated in Figure 2, show the ratio of the "true positive" and "false positive" samples in classification when the threshold for each binary one-vs-rest SVMclassifier score is changed. The area under the ROC curve (AUC) is related to the fidelity of the classification result. The AUCs for the ROC curves were: class 1 (extensive tubule formation) vs. classes $\{2,3\}, 0.84$; class 2 (moderate 


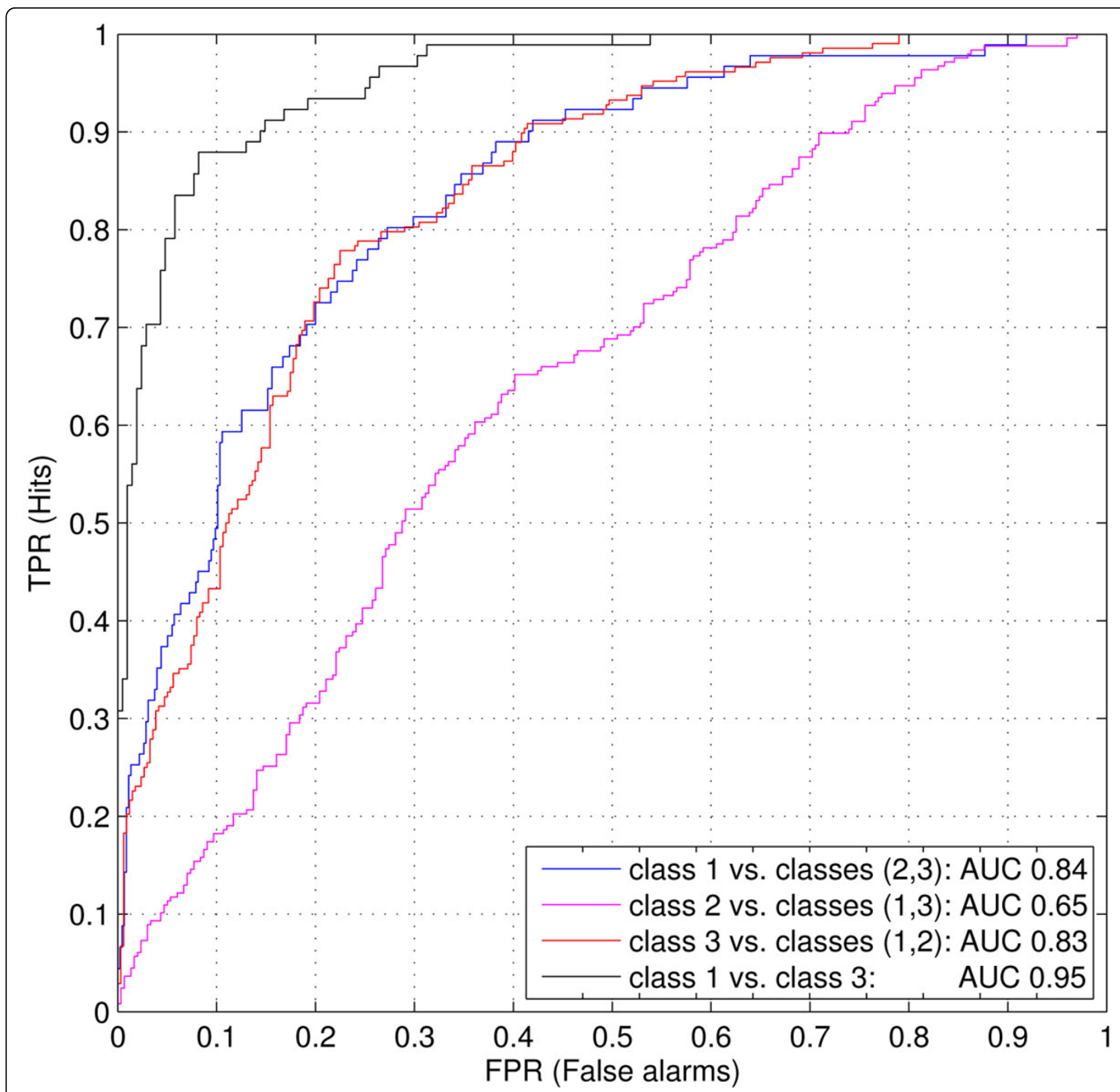

Figure 2 ROC curves showing the classification performance Receiver operating characteristic (ROC) curves for each of the three one-vs-rest SVM classifiers. Fourth ROC curve is for an SVM classifier separating classes 1 and 3. Also the relative areas under the ROC curves (AUC) are denoted which express the fidelity of the classification result.

tubule formation) vs. classes $\{1,3\}, 0.65$; and for class 3 (no tubule formation) vs. classes $\{1,2\}, 0.83$. If each image is classified into the class with the highest SVM score, the total classification accuracy is $62.0 \%$. The total classification accuracy was improved by $2 \%$ by using the LPQ descriptor in addition to the traditional LBP descriptor. It seems that the separation of intermediate class 2 from the classes 1 and 3 is the most challenging task. This is understandable since image content in class 2 samples is a mixture of the two neighbouring classes 1 and 3 . If it would be enough to separate only the extreme morphological classes 1 and 3 neglecting the class 2, a single binary SVM classifier could be used. For this class 1 vs. class 3 classifier AUC is 0.95 which is remarkably better than the results for the one-vs-rest classifiers. The accuracy of class 1 vs. class 3 classifier is $90 \%$ (when threshold $=0$ for SVM score is used). One option for better separation of class 2 could be to do the analysis for smaller image areas which would be classified as class 1 or 3 . Then class 2 could be found as an appropriately selected mixture of these areas. 


\section{Conclusions}

Histological grade of breast cancer is regarded as an important prognostic factor, but not included in staging guidelines due to the subjective nature of the assessment process. In the current study, we propose a computer vision method based on texture features and a classifier utilizing supervised machine learning to discriminate between cancer morphology as determined by a human observer. The results obtained show that automated grading is feasible and that discrimination between different levels of tubule formation can be performed with moderate to high accuracy. By combining LBP and LPQ features it is possible to improve the discrimination accuracy compared to using only LBP alone. While the extreme morphological structures according to tubule formation in the breast cancer tissue are discriminated with high accuracy, the recognition of the intermediate class should still be improved.

\section{List of abbreviations used}

AUC: Area under the ROC curve; HE: Hematoxylin-eosin; LBP: Local binary pattern; LPQ: Local phase quantization; RBF: Radial basis function; ROC: Receiver operating characteristic; SVM: Support vector machine; TMA: Tissue microarray

\section{Competing interests}

The authors declare that they have no competing interests.

\section{Authors' contributions}

VO designed, implemented, and tested the classification system and wrote related parts of the article. NL annotated and labeled the images used in the experiments and wrote the medical sections of the article. ER consulted significantly for the design of the algorithms. MP consulted for the design of the system. ML was responsible for handling and preprocessing of image data in the virtual microscope environment. HJ was the principal investigator of the FinProg study. JL was responsible for designing the multidisciplinary research setting as a whole. ER and JL contributed also by improving the article. All authors read and approved the final manuscript.

\section{Acknowledgements}

The study was kindly supported by the national Biomedinfra and Biocenter Finland projects.

\section{Authors' details}

${ }^{1}$ Institute for Molecular Medicine Finland-FIMM, Helsinki, Finland. ${ }^{2}$ University of Oulu, Center for Machine Vision Research, Oulu, Finland. ${ }^{3}$ Department of Oncology, Helsinki University Central Hospital, Helsinki, Finland.

Published: 30 September 2013

\section{References}

1. Elston CW, Ellis IO: Pathologic prognostic factors in breast cancer. I. The value of histological grades in breast cancer. Experience from a large study with long-term follow-up. Histopathology 1991, 19:403-410.

2. Singletary SE, Allred C, Ashley P, Bassett LW, Berry D, Bland KI, Borgen PI, Clark G, Edge SB, Hayes DF, Hughes LL, Hutter RVP, Morrow M, Page DL, Recht A, Theriault RL, Thor A, Weaver DL, Wieand HS, Greene FL: Revision of the American Joint Committee on Cancer staging system for breast cancer. J Clin Oncol 2002, 20(17):3628-36.

3. Boiesen P, Bendahl PO, Anagnostaki L, Domanski H, Holm E, Idvall I, Johansson S, Ljungberg O, Ringberg A, Ostberg G, Fernö M: Histologic grading in breast cancer: reproducibility between seven pathologic departments. In Acta Oncol. Volume 39. South Sweden Breast Cancer Group; 2000:(1):41-5.
4. Lundin M, Lundin J, Isola J: Virtual microscopy. J Clin Pathol 2004, 57:1250-1.

5. Linder N, Konsti J, Turkki R, Rahtu E, Lundin M, Nordling S, Haglund C, Ahonen T, Pietikäinen M, Lundin J: Identification of tumor epithelium and stroma in tissue microarrays using texture analysis. Diagn Pathol 2012, 7:22.

6. Dalle JR, Leow WK, Racoceanu D, Tutac AE, Putti TC: Automatic breast cancer grading of histopathological images. Conf Proc IEEE Eng Med Biol Soc 2008, 3052-5.

7. Doyle S, Agner S, Madabhushi A, feldman M, Tomaszewski J: Automated grading of breast cancer histopathology using spectral clustering with textural and architectural image features. IEEE International Symposium on Biomedical Imaging: From Nano to Macro 2008, 29:496-499.

8. Rahtu E, Heikkilä J, Ojansivu V, Ahonen T: Local Phase Quantization for Blur-Insensitive Image Analysis. Image and Vision Computing 2012, doi: 10.1016/j.imavis.2012.04.001, Matlab code database [http://www.cse.oulu.fi/ CMV/Downloads/LPQMatlab].

9. Ojala T, Pietikäinen $M$, Mäenpää T: Multiresolution gray-scale and rotation invariant texture classification with local binary patterns. IEEE Transactions on Pattern Analysis and Machine Intelligence 2002, 24(7):971-987, Matlab code database [http://www.cse.oulu.fi/CMV/Downloads/LBPMatlab].

10. The FinProg breast cancer database. [http://www.inprog.org].

doi:10.1186/1746-1596-8-S1-S29

Cite this article as: Ojansivu et al:: Automated classification of breast cancer morphology in histopathological images. Diagnostic Pathology 2013 8(Suppl 1):S29.

\section{Submit your next manuscript to BioMed Central and take full advantage of:}

- Convenient online submission

- Thorough peer review

- No space constraints or color figure charges

- Immediate publication on acceptance

- Inclusion in PubMed, CAS, Scopus and Google Scholar

- Research which is freely available for redistribution 